\title{
Understanding the user perceptions of the public and private threshold in intensive housing design in Wellington, New Zealand
}

\author{
Ariana Faulkner ${ }^{1}$, Nilesh Bakshi ${ }^{1,2}$, Fabricio Chicca ${ }^{1}$, Andre Brown $^{1}$ and Frances Moughan ${ }^{2}$ \\ ${ }^{1}$ Victoria University of Wellington, Wellington, New Zealand. \\ ${ }^{2}$ Studio of Pacific Architecture. \\ arianafaulkner1997@gmail.com; Nilesh.Bakshi@vuw.ac.nz.
}

\begin{abstract}
This study investigates user perception of the public and private threshold within intensive housing in Wellington, New Zealand. The specific focus is to investigate the perception within the threshold that is the transitional area between the front door and the street curb. Two Wellington, New Zealand, based housing developments, The Altair complex (Newtown) and Britomart complex (Berhampore), were selected as case studies against specific criteria. These criteria promote a diverse target demographic to increase variability in results. Human experience within the public and private threshold are recorded and collated through a series of semi-structured interviews. The objective of the study is to understand the residents' personal opinion on the space; what works and what doesn't. The results are analysed and relevant literature is integrated to reveal trends and design implications. Ultimately, a set of guidelines and design implications are produced, which can be followed at a design level. These strategies aim to guide the design-phase in future industry projects, therefore, improving the human experience within intensive housing.
\end{abstract}

Keywords: User perception, public and private threshold, intensive housing, medium-density housing.

\section{Introduction}

This research investigates user perceptions of the public and private threshold space within New Zealand intensive housing. To this end, the opinions of residents were collected in order to understand how dwelling occupants perceive their thresholds between public and private space. To understand definitions of successful transitions between public to private space this research adopted an interview based data collection method to examine how intensive housing residents could inform key design guidelines for practitioners.

The aim of this research was to produce a set of guidelines and principles based solely on user perception. The qualities that influence human experience is critically analysed to create correlations between the user experience and the public and private threshold.

Revisiting the Role of Architecture for 'Surviving' Development. $53^{\text {rd }}$ International Conference of the Architectural Science Association 2019, Avlokita Agrawal and Rajat Gupta (eds), pp. 481-490. (C) 2019 and published by the Architectural Science Association (ANZAScA). 
Occupants in two recently completed intensive housing projects in Wellington, New Zealand, dubbed 'The Altair' and 'Britomart', were interviewed. Semi-structured interviews were recorded onsite at the case study locations to understand correlations between user groups and locations. Then the interviews were analysed to understand the effects of the threshold and revealed common trends. The following section speaks to the specific data collection and analysis method adopted in the interviews carried out for this research.

\section{Methodology}

To find the most relevant case study projects for this research, a statistical analysis of all surrounding suburbs of the Wellington Central Business District was carried out. This initial statistical analysis of the 2013 census data recorded (the most updated data available at the time of this research). This data included variables such as occupants' gender, age range, ethnic group, housing typology, median weekly rent and, median income. Factors such as housing typologies of the suburbs were also taken into account, suburbs with high rates of medium/high-density living were favored. This information briefed the research on what to expect in each suburb. The objective of the research was to gain a range of user perceptions, therefore, suburbs with the most diverse range of the aforementioned variables were selected for inclusion in this research.

As a result two suburbs, Newtown and Berhampore, were chosen for their diverse range of households matching the original aim of the research. Both neighborhoods are within walking distance to the city centre. This creates a spectrum of transport needs and variations for users. This close proximity to the city centre or 'semi-suburban' location also means that it is used as a 'thoroughfare' suburb, not an end destination. As a consequence, this has created a large amount of diversity in these two suburbs, and meeting the criteria for the initial case study scheme.

\section{Prerequisite case study analysis}

\subsection{The Altair}

\begin{tabular}{l|l}
\hline Location & $108-126$ Rintoul Street, Newtown, Wellington \\
\hline Year completed & 2006 \\
Architect & Architecture+ \\
Number of dwellings & 71 total \\
Building typology & Row style terrace houses (2, 3 and 4 storey townhouses) \\
Site Size & Approximately $100 \mathrm{~m} \times 100 \mathrm{~m}$ (1 hectare) \\
Site density & Each individual site has an area of between $130 \mathrm{~m}^{2}$ and $180 \mathrm{~m}^{2}$. \\
& Averaging $142 \mathrm{~m}^{2}$ excluding courtyard. \\
\hline
\end{tabular}

In order to evaluate the phenomenological qualities of each case study, the architectural design qualities are described here in detail based on the architect's design brief publications. The Altair Townhouses are arranged around two large courtyards to enhance community interaction. Modulations in form, materiality and, color produce a sense of identity for each of the dwellings (Figure 1). This is achieved by staggering the unit sizes to create an

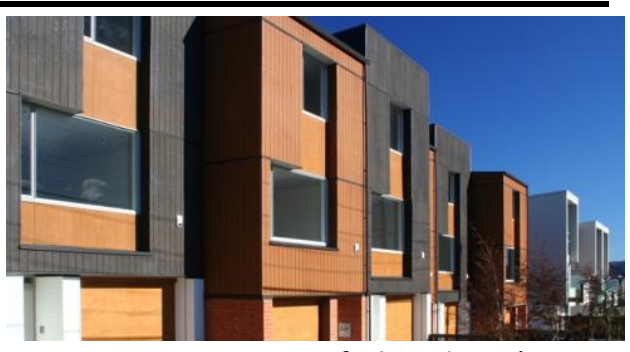

Figure 1. Street view of The Altair (source: Architecture+, 2019) 
irregular aesthetic. The aim of this irregular aesthetic was to provide a greater degree of privacy between units. In order to add to this sense of individual identity, there is also a large range of urban modulation; vehicle access, private courtyards, common areas and foliage to distinctively separate spaces. Three larger communal landscape areas within the complex create the main separations between the three groups of buildings (Figure 3).

Two housing blocks have a street-front facade on the North and east ends of the complex. These two street-facing housing blocks are separated by a vehicle entry and exit zone. The two housing blocks have different conditions:

1. Parking access from the rear and a semi-private gated courtyard with direct pedestrian access from the street. (Figure 2)

2. Parking and access from Rintoul Street and private courtyards at the rear.

GROUND LEVEL FLOORPLAN TO STREET FABRIC (UNIT 13)

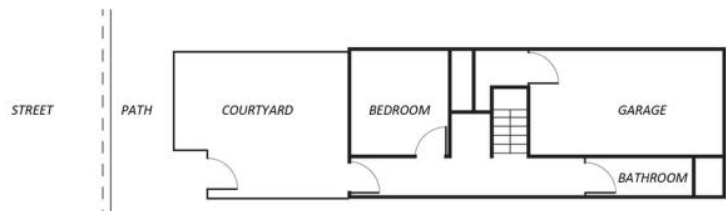

Figure 3. Plan
MASTERPLAN OF THE ATLAIR

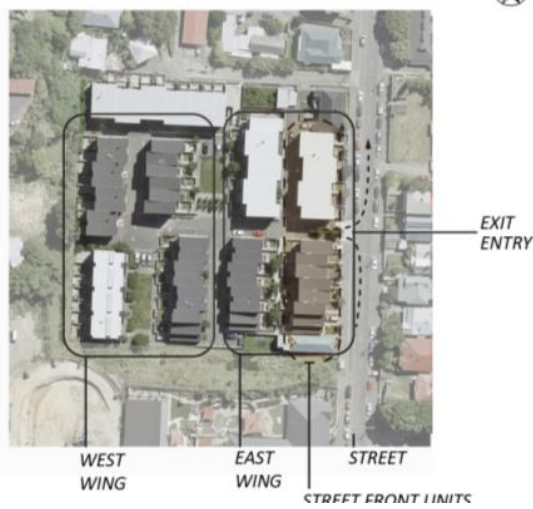

Figure 2. Masterplan (source: google maps, 2019)

\subsection{Britomart}

\begin{tabular}{ll}
\hline Location & 135 Britomart Street, Berhampore, Wellington \\
\hline Year completed & 2018 \\
Architect & Studio Pacific Architecture \\
Number of dwellings & 36 units in total \\
Building typology & Terraced Housing \\
Site Size & $2023 \mathrm{~m}^{2}$ \\
Site density & - \\
\hline
\end{tabular}

Britomart housing development is built to serve the social housing demand for smaller apartment-style homes in Wellington. The development has been designed with a community focus, whilst still maintaining privacy for residents. All homes are northfacing, to attain maximum sunlight all year round (Figure 4). Ground floor units are all accessed by a small

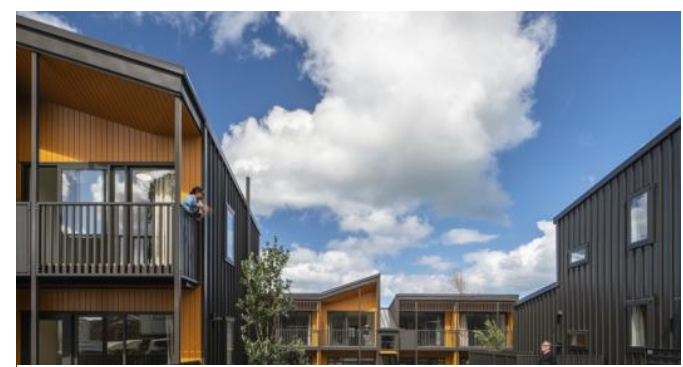

Figure 4. View of Britomart from street (source: Studio Pacific Architecture, 2018) 
private fenced patio which has built-in planter boxes and clotheslines (Figure 5).

Level 1 units are accessed by a shared entry staircase, creating a sense of community within the entranceway (Figure 6). On-site parking is minimised to favour a pedestrian-based landscape. This is a response to the close proximity to local amenity and public transport routes.

GROUND LEVEL FLOORPLAN TO STREET FABRIC (UNIT 6)

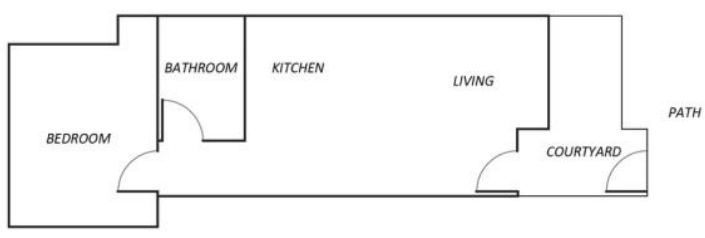

Figure 5. Plan

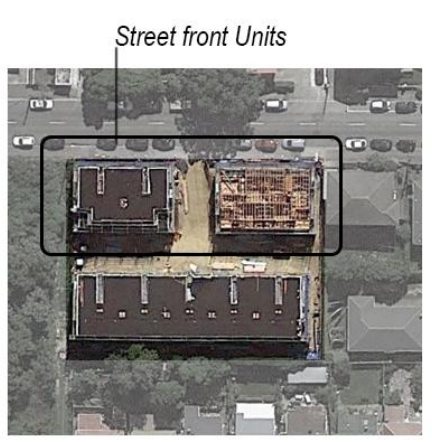

Figure 6. Britomart Masterplan (source:

google maps, 2019)

\section{Interview procedure and pilot study interviews}

The work carried out in this research followed the Human Ethics Committee (HEC) guidelines from Victoria University of Wellington and subsequently attained ethics approval [0000027137]. A semistructured interview method was used for the study. Open-ended questions that relate directly to the design qualities were used to create space for participants to narrate their experiences (Galletta \& Cross, 2013). This was because of the unprecedented nature of this research which is based on the methodology developed by Kernohan et al. (1996). As a consequence, the biggest obstacle was establishing a research methodology that would facilitate clear communication between all members of the research team and the interviewees. This was because the user perception survey developed here brought together practice based designers, academic researchers and residents with little or no experience in design or research.

The methodology discussed in the following section discusses how Kernohan et al's work is applied to the aforementioned case studies. Kernohan et al. address the topical issue of user participation. At the time of its publication this work was identified as a major addition to the literature of user studies and building evaluation (RIBA, 1996).

\subsection{Pilot Study Interviews}

The aim of the pilot study interviews was to obtain at least 10 interview responses from occupants who had been occupying a dwelling in each case study. Two interview groups were put together with research assistants and architects from the industry partner. A total of eleven interviews were recorded in the initial phase of the interview period, throughout this phase, various changes were made to the question framework and terminology to improve legibility and communication between design experts, the researchers and the dwelling occupants.

The line of questions were structured specifically to increase the interviewees understanding of the research topic. Questions based on Spatial Qualities and Recreation were posed at the very 
beginning of the survey to establish key definitions of public and private thresholds. In nearly all interviews this evoked more thought-provoking topics such as occupants' privacy needs, which lead to informed critical feedback. These opening questions also allowed the research and design team to gauge an understanding of the user's attitudes towards thresholds at an early stage of the interview process. This was one of the major findings of the interview process which is discussed in greater detail at the end of this paper in preparation of phase two of this research.

\subsection{Initial Interview questions}

\section{Space Qualities}

a. Do you enjoy being able to personalise your outdoor area?

\section{Recreation}

a. What activities do you like to do in your outdoor area?

b. Are there any activities you would like to do in your outdoor area but can't?

c. How often do you use the communal facilities of the complex?

\section{External Environments}

\section{Community}

a. Would an external space need any particular shelter? (From wind, rain or sun?)

a. Do you like to be able to easily communicate or socialize with your immediate or close neighbours?

b. Would you like to live in an integrated way with families and other demographic groups in neighbouring dwellings?

\section{Public and Private Environments (privacy needs)}

c. Is it important to you for have privacy from the other units and/or road?

d. Does it bother you that people or other units can see into your outdoor space?

e. Would you rather have more sun or more privacy?

f. Does the public see through to this dwelling area? How does this affect you?

The two case study developments were then interviewed on separate days:

The Altair: 8 residents interviewed between the 31/01/19 - 1/02/19

Britomart: 3 residents interviewed on the $7 / 02 / 19$

\section{Interview results}

\subsection{The Altair}

\subsubsection{Spatial Qualities}

The interview responses suggest that $75 \%$ of residents like to personalise the space to their needs. Specifically, resident 1 identified that personalisation is not necessary because of the tenure. A key point of fact here was that this resident was renting the property and therefore preferred to maintain the original state of the area. This resident also identified that if the property was privately owned the area would be well-tailored to his own preference. This was a common theme amongst two other residents, who agreed with this sentiment; essentially reiterating that they would consider more personalisation if the property was privately owned. Resident 6 specifically identified being dissatisfied with thresholds between public and private space because of the lack of sunlight near these areas. This 
resident specifically noted not spending "any time" there. This was not a common theme and seemed to be an isolated response

\subsubsection{Recreation}

All residents do functional and recreational activities in their outdoor area including drying clothes, gardening, sitting, eating meals, drinking, and playing with children. Resident 7 specifically emphasised spending a vast amount of time in the outdoor area because of the access to sunlight.

As a result, and as could be expected, all the instances that residents identified feeling dissatisfied with their threshold space had a direct correlation with either a lack of sunlight in the outdoor areas, frustration with building layout and lack of space for entertaining guests.

When asked about the communal residents' park amenities, one resident vehemently responded in favour of this kind of space, stating that their family used it "almost every day" since it is enclosed and feels safe for children. This use was expected by both the research team and the architects as initial research in the planning stage identified that children are by far the greatest users of shared outdoor space in multi-family housing (Marcus, 1986, p. 126). Multiple units overlook the residents' park so there is passive visual surveillance for children playing. Conversely, all residents who identified they did not use this communal park noted that there was no amenity for adults in this space, making it unattractive for anyone without children.

\subsubsection{External Environments}

The majority of residents (75\%) noted that the inclusion of rain shelter is necessary for any threshold spaces. Resident 3 notes their displeasure with the lack of weather-protection over the balcony, stating that the potential risk for slips in wet weather condition meant that these spaces were often unusable or did not feel safe. The residents located towards the west wing of the complex were all satisfied that these external thresholds spaces were "fairly protected as it is" due at least in part, to the added protection of the shelter against the north-easterly down pours (NIWA, 2014, p.16)

\subsubsection{Community}

With the exception of a single resident, all interviewees identified that they liked the ablility to socialise with immediate or close neighbours. A resident with children specifically noted that:

"The kids play with other children in the area and it's absolutely amazing, it's a great way of meeting new people".

This suggested that incorporating additional spatial consideration for social space was potentially important, especially for households with children. All of the residents that were located in the East wing of the complex (street-front side) identified that they were not open to living in a more integrated way with families and other demographic groups in neighbouring dwellings. One resident specifically identified that they were "happy the way it is". However, Resident 3 suggested they would be open to such a concept but not in the current complex. The conversation with the design team during the interviews suggested that this was potentially due to their location in the complex. These residents were relatively cut off from the central part of the complex simply because their units had dedicated street front access. All accounts of the residents interviewed suggested that any residents located near the street had less daily interaction with other residents. However, this may have been a result of the choice made not to integrate with neighbouring dwellings and households made up of families.

\subsubsection{Privacy}

All residents located on the East wing of the complex (street-front side) agree that privacy from the other units and/or road is of a medium/high priority. Resident 2 identified that noise pollution from 
the road was a big factor in this decision. This resident identified being extremely unhappy with the current visual and audio privacy, specifically stating that that the public and private threshold should be more than just a visual aspect. All other residents that did not have street-front dwellings suggested they were satisfied with their current privacy from other units/road, with four out of five residents stating that they enjoyed having a unit located away from the street fabric. Resident 7 explained that their satisfaction with privacy is based predominantly on safety reasons; they stated that the outdoor area wouldn't be used if their unit was situated near the road, due to perceived safety issues for children. These results study suggest there is a clear difference in privacy satisfaction between users. The significant difference in the level of satisfaction had to do with the location of the dwelling, the preferred option being situated away from the road.

Around $66 \%$ of residents situated near the street-front rated privacy needs higher than sunlight needs. The remaining residents all rated sunlight higher than privacy. Again the difference in privacy needs seemed to be associated with the location of the unit.

\subsection{Britomart}

\subsubsection{Spatial Qualities}

The interviews suggest that the residents would like to be able to personalise their outdoor space with gardening being proposed as a desirable activity.

\subsubsection{Recreation}

All residents thoroughly enjoyed recreational and functional activities in their outdoor thresholds. These included gardening, sitting, reading and be able to dry clothes. Resident 11 described the merits in having a raised garden bed in the outdoor area, identifying that this made it much easier to reach the garden.

To improve recreational aspects, all residents proposed having space in their external threshold spaces to grow more vegetables and more space to entertain. Amongst all the interviewees, the data collected in this survey suggested that gardening was a great success for the older demographic.

All residents identified that they were not using the communal facilities of the housing complex. Two residents explained they did initially use this space when they moved in, however the configuration of this space within the complex made it increasingly inconvenient over time. The results of this survey also suggest that the access to the drying room is not adequate as it is too steep for the elderly residents.

\subsubsection{External Environments}

Much like the Altair complex all the residents located on the street-front row are exposed to a lot of wind. The household in these space all identified shelter from wind was the biggest problem here.

\subsubsection{Privacy}

Privacy from the other units and/or road was again identified high priority for residents on the street-front row, and some specific issues with privacy were noted. Some outdoor areas are overlooked by neighbouring dwellings; which was specifically identified as an undesirable attribute in the building layout by all interviewees. The permeability of the main entrance of each dwelling was also identified as an inappropriate design measure. This was because the main doors were partially clear glass, as a result, pedestrians are constantly able to see into the internal private dwelling spaces. 
Although not all residents expressed concern regarding people/other units being able to see into their space, all household identified that they needed a greater degree of privacy.

\section{Common trend analysis}

The results of this survey identify six common trends which were observed in both case studies. These centrally focus on dwelling location within the masterplan; lack of privacy and permeability of the building envelope; lack of entertainment space in the external threshold spaces; the massive discrepancy in user satisfaction between households facing the street front in comparison to households facing inwards in the complex; the social interactions inhibited by the communal space design; and finally noise pollution. These are discussed in greater detail below.

1. Majority residents have a shared level of satisfaction with their threshold irrespective of their demographic. However, this satisfaction was lower within the residents located close to the road. The investigations suggests that privacy is a concern that has been poorly addressed in those houses. Residents in this area noted that they would add more privacy layers to the boundary if they owned the property. Privacy needs are higher for the residents living on the street edge, with all respondents in this zone saying privacy is of a medium to high priority. $80 \%$ of residents situated on the street-front rate privacy needs as higher than their need for direct sunlight. Residents living in other areas noted that they are pleased with the location of their dwelling. Some users stating that their current privacy is "great" and they are "lucky" not to live near the street. All residents in these areas rated direct sun need higher than privacy needs.

2. Interviewees voiced concerns about people/the public being able to see into their outdoor space for residents living on the street front edge. This is compared to there being little or no irritation from the public seeing into outdoor space for the residents located to the rear-side of the complex.

3. Residents are dissatisfied with the outdoor area in terms of places for entertaining. Residents noted that if the outdoor area was connected to the living space they would use it much more often. This suggests the willingness to blur the threshold between private and public; residents want to bring social aspects into their outdoor area (bring their private life towards the public sphere).

4. Residents who live on the street front typology are less inclined to live in a more integrated way with families and other demographic groups in the area. The occupants of dwellings with street front access have little incentive to enter the housing complex to interact with neighbours or use the common areas. As Marcus (1986) explains a greater territorial sense can develop if residents frequently walk through communal spaces. Residents situated at the rear of a complex may begin to feel comfortable in the space, greet others and perceive the space almost as an extension of their dwelling (Marcus, 1986, p.120). To create a more interactive community, there needs to be more incentive for street-front residents to enter the complex.

5. Some residents have noted that there are little communal facilities available to promote social encounters. The lack of outdoor communal amenities for adults was apparent from the interviews. According to Marcus (1986), most people will avoid private ground level open spaces lacking a barrier and abutting directly onto public spaces (Marcus, 1986, p. 127). The results of this research suggest that the threshold between private to public is unsuccessful. The common area is shared by all residents, yet the area is underutilised. This area needs more amenities to invite users to enjoy the space, especially street-front residents who have no incentive to enter the space. 
6. A small number of residents on the street-front have explained that noise pollution from the road matters just as much as privacy. Not only visual privacy is needed but also sound privacy. Noise pollution problems were only mentioned by street-front users; meaning this may be of a high priority for a successful threshold into a neighbourhood.

\section{Design Implications}

The data collected in regard to the public-private threshold in this study identifies that secluded dwellings were perceived as far more successful. Specifically the units that bordered the existing street were perceived as far less successful at creating appropriate public to private thresholds. Perceptions of residents located on the street front were much more negative compared to those who were located deeper in the complex.

Participants particularly noted the lack of barriers from road noise pollution. Natural barriers such as landscaping is recommended to dissipate not only the acoustic but also visual pollution.

Where the public-private threshold extended to the treatment of facades and point of entry, users identified that screening or frosted glass would be preferred over a fully transparent material.

A common trend was the opinion that if the property were privately owned by the resident (rather than rented) or if a sense of ownership was afforded then there would likely be more motivation for households to develop vegetation and landscaping in the outdoor areas. This was specifically identified by some interviewees as a measure that could have potentially added more privacy from other units and the road. Nearly all interviewees gave landscaping a very high priority in their outdoor area; multiple residents commented on how "lucky" they are to have a tree in their outdoor area. Therefore, the use of landscaping to increase the feeling of privacy and ample room for gardening would be recommended as identified by Marcus (1986).

Some residents also identified being unhappy with their threshold in places where units had balconies physically overlooking their private courtyard. The interviews found that the majority of residents were uncomfortable with other resident's looking down into their private space. Desired levels of privacy vary according to the individual. This suggests that designing for inclusion and flexibility may be one of the most important consideration of design thresholds in intensive housing projects.

\section{Barriers and limitations}

It should be noted that this research is not without limitations. First, this paper only looked at the residential sector based on the households examined in two case studies. While this study is a comprehensive representation of a wide range of ethnic groups in the case studies examined, it is still limited due to the single type of residential housing examined. Second, the data collection was limited by the availability of the residents. As interviews took place between $11 \mathrm{am}-4 \mathrm{pm}$ on weekdays there were a number of households that were not home. Involvement in this study was also governed by the residents' willingness to participate, and times of availability which were dictated by day time and early evening hours for the safety of the research team. Third, this research is based in specific suburbs of Wellington, New Zealand. The contextual nature of qualitative research means that careful thought must be given to the potential relevance (or lack of) to other sociocultural settings (Kuper \& Lingard \& Levison, 2008). 


\section{Key outcomes and further research}

Some of the most lessons learnt in this research pertain to the manner in which this kind of phenomenological research can be undertaken again with a wider study-pool. One of the key outcomes of this research was the iterative evaluation of the survey methods that were refined during the pilot study and then in the final questionnaire. As a result, the exercise of developing a user perception survey has been reported on earlier in this paper.

The biggest obstacle in this research was facilitating research between all members of the research team and the interviewees. This was because of the unprecedented nature of this research that was initially proposed by the methodology developed by Kernohan et al. (1996) over two decades ago. The user perception survey developed here brought together practice based designers, academic researchers and residents with little or no experience in design or research. This meant that industryrelated jargon and research based definitions had to be calibrated to develop a common terminology for all the groups in question. The questions were then arranged into a simple framework with clear themes to promote a coherent structure and communication. In partnership with Victoria University of Wellington and Studio Pacific Architecture this 'user perception survey' will now be the main tool used for understanding perception of occupants in the next larger phase of the study.

\section{References}

Architecture+. (2019). Altair [Image]. Viewed on 2 July 2019 <http://architectureplus.co.nz/multi/altair/>

Galletta, A. \& Cross, W. E. (2013). The Semi-Structured Interview as a Repertoire of Possibilities. In Mastering the Semi-Structured Interview and Beyond. 45-72: NYU Press.

Google Maps (2019). Google Maps [online] Sateliette view, viewed 2 July $2019<$ https://www.google.com/maps/@$41.3158279,174.7773586,223 \mathrm{~m} /$ data $=! 3 \mathrm{~m} 1$ !1e3>

Kuper, A., Lingard, L.\& Levison, W. (2008). Qualitative Research: Critically Appraising Qualitative Research. BMJ: British Medical Journal, 337, 687-689.

Marcus, C.C. \& Sarkissian, W. (1986). Housing as if people mattered: site design guidelines for mediumdensity family housing / Clare Cooper Marcus and Wendy Sarkissian; with Sheena Wilson and Donald Perlgut.

Marcus, C.C. \& Francis, C.A. (1998). People places: design guidelines for urban open space / edited by Clare Cooper Marcus and Carolyn Francis; drawings by Su Sin Tang and Yun Flora Yeh. 2nd ed., New York: Van Nostrand Reinhold.

Studio Pacific Architecture. (2018). Te Maru O Tawatawa Britomart Street Social Housing. <https://www.studiopacific.co.nz/projects/2018/te-maru-o-tawatawa-britomart-street-social-housing/>

KERNOHAN, D., GRAY, J., \& DAISH, J. (1996). User participation in building design and management: a generic approach to building evaluation. Oxford, Butterworth Heinemann. http://catalog.hathitrust.org/api/volumes/oclc/34547473.html.

Wardle, J. (2002). Developing Advertising with Qualitative Market Research. The Limitations of Qualitative Research. SAGE Publications, Ltd, London. 